\title{
A SPECIAL-PURPOSE PROGRAM FOR EARTHQUAKE LOCATION WITH AN ELECTRONIC COMPUTER
}

\author{
By John M. Nordquist
}

\begin{abstract}
An electronic digital computer program has been developed for determining the source and origin time of a local earthquake by the method of least squares, using the times of arrival of direct and refracted $P$ waves at stations in the Pasadena network. Output includes the geographic coordinates and depth of the source, the origin time, direct distances from the source to each station, and the difference between observed and computed arrival time of $P$ at each station. Limitations to the applicability of the program are discussed.
\end{abstract}

\section{INTRODUCTION}

The use of high-speed digital computers for processing seismic data is increasing rapidly. Starting in August, 1960, the U. S. Coast and Geodetic Survey has used an electronic computer for routine location of the larger earthquakes recorded throughout the world. Bolt (1960) has developed a program for the revision of epicenters by the method of least squares, using up to 300 observations of $P, p P$, and $P K P$. This program takes advantage of the large storage space and high speed of the IBM 704 computer. The Australian National University (Flinn, 1960) is using an IBM 650 computer for determining epicenters of earthquakes in New South Wales, with computation limited to the use of arrival times of direct $P$ phases at stations in that region.

The Seismological Laboratory in Pasadena determines routinely the locations and magnitudes of all earthquakes in the southern California area which are well recorded by the stations of its network, except for aftershock sequences in which the number of such shocks becomes too great to handle and the magnitude threshold must be raised. In the past, these determinations have been made by graphical methods, with numerical checks on the consistency of the results. The routine load has averaged twenty local earthquakes of magnitude 3 or over per month. Special research has been done on the distribution of epicenters in some aftershock sequences, but there remains a large backlog of data on aftershocks which has not been interpreted because of limitations of time. The purchase of a Bendix G-15D electronic digital computer has made possible the application of the method of least squares to the determination of epicenters not only for special investigation of particularly interesting earthquakes, but also to the routine load and to aftershock swarms as well.

The program described here was designed for the Bendix G-15D by the author to permit use of data from the 17 permanent stations of the Pasadena network and others up to a maximum of 24 , to apply travel-times of refracted as well as direct $P$ phases as determined for the region, and to operate with a minimum of supervision on the part of the computer operator, so that computation may proceed unattended overnight. At the same time, features have been included which permit optional typeout of station residuals after each iteration, and deletion of unsatisfactory station data or alteration of station weights and $P$ phase identifications. 


\section{Operating Procendure}

Data input is by punched paper tape prepared off-line on a Flexowriter. For each earthquake, approximations to the epicenter and origin time are given, followed by the times of arrival of the first phase at the various stations of the Pasadena network, the weights assigned to the stations, and, if desired, specification of the $P$ phase at each station. This data is read into the computer automatically one set at a time. The program edits the data to remove any obviously erroneous entries and types out the data which remain. It then applies the method of least squares to compute corrections to the coordinates of the focus and to the origin time.

The least-square solution is repeated for the corrected location and origin time until the correction to the location is less than a pre-specified amount, or until a pre-specified number of iterations has been performed. The geographic coordinates of the corrected location are then typed out, followed by a listing of the direct distance of the source from each station (for magnitude computation) and the difference between observed and computed arrival times for the $P$ phase, and the standard errors of determination of the coordinates and of the origin time.

This sequence of operations is performed on each set of data in turn until the end of the tape is reached, whereupon the computer comes to a halt.

The program is in machine language, using the arithmetic and output subroutines of Autopoint 24, in which all numbers appear in the machine as double-precision binary numbers scaled $2^{-24}$. The arithmetic subroutines scale the results automatically to $2^{-24}$. A multiplication or division requires $0.09 \mathrm{sec}$, and a square root is extracted in 0.25 sec. The output subroutine converts a number from the binary form and types it out as a decimal.

The entire program, including storage locations for input data and results of intermediate calculations, is accommodated in the 2,160 -word magnetic drum memory of the computer.

A solution requiring three iterations, using the data from 13 stations is completed in nine minutes. This indicates that the routine load of local earthquakes will require three to four hours of machine time per month.

Figure 1 shows the complete typeout during the location of one earthquake, with explanatory notes added in parentheses.

\section{Traveu-Times}

Travel-times of $P$ phases are based on an assumed three-layer model of the earth's crust. The effect of curvature of the earth's surface is negligible for the distances involved. For sources in the upper layer of the crust, the travel-times are given by

$$
\left.\begin{array}{l}
P_{1}-O=D / V_{1} \\
P_{2}-O=\Delta / V_{2}+K_{2}+C_{2} h \\
P_{3}-O=\Delta / V_{3}+K_{3}+C_{3} h
\end{array}\right\}
$$

where $\left(P_{1}-O\right),\left(P_{2}-O\right),\left(P_{3}-O\right)$ are the travel times for paths whose lowest points are in the first, second, and third layer, respectively.

$V_{1}, V_{2}, V_{3}$ are velocities of $P$ in first, second and third layers 
$D \quad=$ distance of focus from station

$\Delta \quad=$ distance of epicenter from station

$K_{2}, K_{3}=$ intercepts of the travel-time curves for a surface source

$C_{2}, C_{3}=$ partial derivatives of the travel times with respect to depth $h$

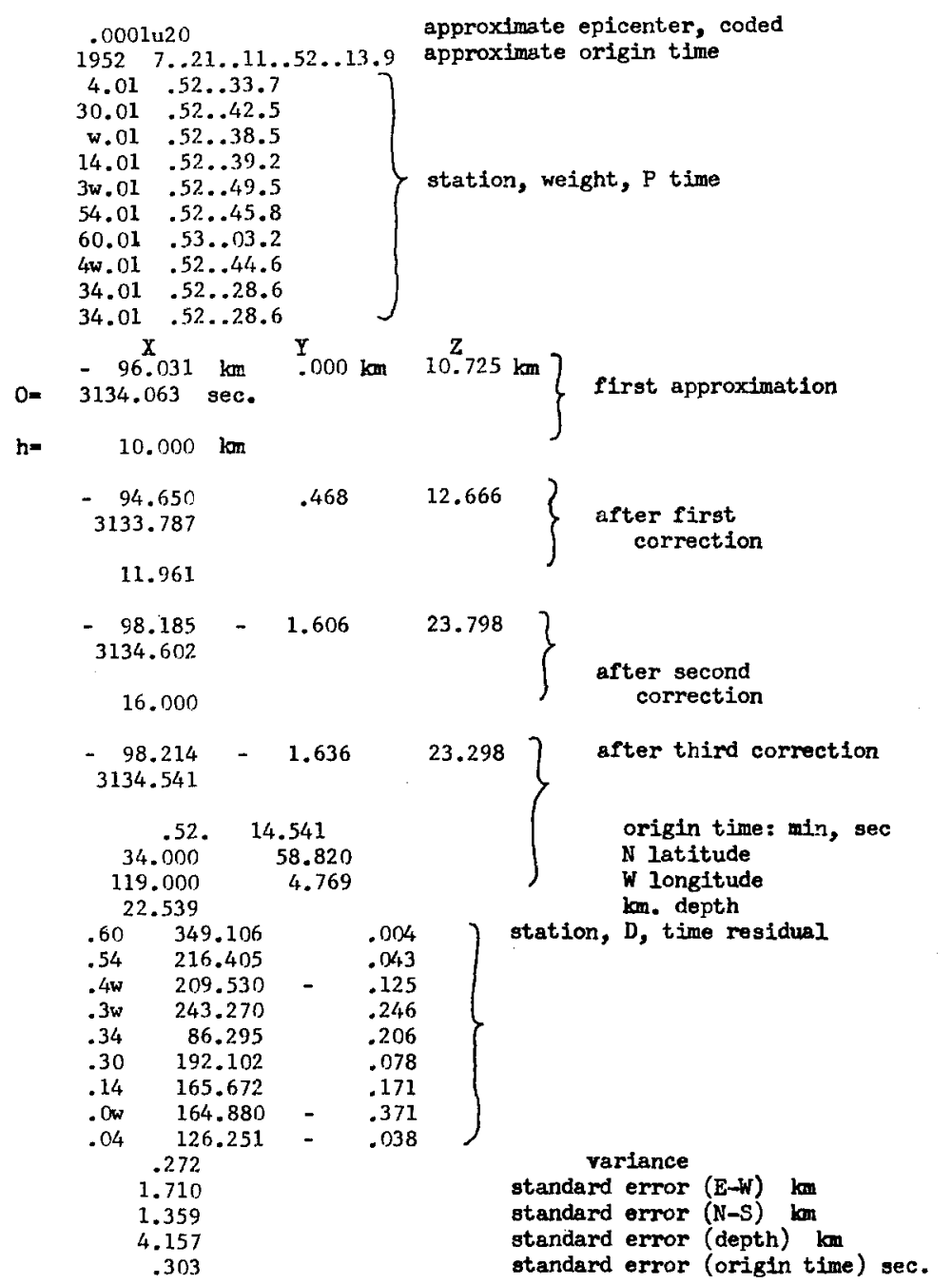

FIG. 1. Complete computer typeout during location of earthquake of 1952 July 21 at $11: 52: 13.9 \pm$ GCT. Note that after the second correction, the corrected depth was below the Conrad discontinuity ( $Z$ of epicenter approximately $0.73 \mathrm{~km}$ ), and that the depth was arbitrarily reduced to $16 \mathrm{~km}$ before the third correction was computed.

Currently the values of the parameters $V$ and $K$ employed in equations (1) are those determined by Press (1960) for the region of southeastern California and Nevada, using explosion data, but other values may be substituted at will.

Past experience suggests that foci of earthquakes in the southern California region usually lie in the upper layer. Calculation of $P_{2}-O$ for sources below the 
Conrad discontinuity appeared to be too complicated to incorporate in the program within the storage limits of the computer. Therefore, if an approximate source is found to be below the Conrad discontinuity, the depth is reduced arbitrarily to bring it into the upper layer before another application of the least-square solution.

\section{Computation}

Computation is carried out in a rectangular coordinate system in which the $X-Y$ plane is tangent to the surface of the earth at $35^{\circ} \mathrm{N}, 118^{\circ} \mathrm{W}$, and the $Z$-axis is directed downward. For each station used, an equation is set up for the corrections $x, y, z$, and $t$ to be added to the coordinates $X, Y$, and $Z$ of the source and the origin time $O$.

Neglecting terms of higher order than the first, these equations are

$$
a_{i x} x+a_{i y} y+a_{i z} z+t=P_{i}-[P-O]_{i}-O=F_{i}
$$

where the subscript $i$ refers to the station

$P_{i}=$ the observed arrival time at the station

$[P-0]_{i}$ is computed from the coordinates of the station and of the approximate source.

In practice, the travel times $[P-0]$ are computed for $P_{1}, P_{2}$, and $P_{3}$ from equations (1) and if no choice of $P$ phase has been specified in the data input, the smallest of the three is chosen. The coefficients of equation (2) are computed using the formulae given in Table 1 for the chosen $P$ phase.

With data from more than four stations the solution is overdetermined and corrections are sought which will minimize the sum of the squares of the residuals $f_{i}$ :

$$
a_{i x} x+a_{i y} y+a_{i z} z+t-F_{i}=f_{i}
$$

If weights $w_{i}$ are assigned to the station data, the desired corrections are the solution of the following equations:

$$
\begin{aligned}
& \sum\left(w_{i} a_{i x}^{2}\right) x+\sum\left(w_{i} a_{i x} a_{i y}\right) y+\sum\left(w_{i} a_{i x} a_{i z}\right) z+\sum\left(w_{i} a_{i x}\right) t=\sum\left(w_{i} a_{i x} F_{i}\right) \\
& \sum\left(w_{i} a_{i x} a_{i y}\right) x+\sum\left(w_{i} a_{i y}^{2}\right) y+\sum\left(w_{i} a_{i y} a_{i z}\right) z+\sum\left(w_{i} a_{i y}\right) t=\sum\left(w_{i} a_{i y} F_{i}\right) \\
& \sum\left(w_{i} a_{i x} a_{i z}\right) x+\sum\left(w_{i} a_{i y} a_{i z}\right) y+\sum\left(w_{i} a_{i z}^{2}\right) z+\sum\left(w_{i} a_{i z}\right) t=\sum\left(w_{i} a_{i z} F_{i}\right) \\
& \sum\left(w_{i} a_{i x}\right) x+\sum\left(w_{i} a_{i y}\right) y+\sum\left(w_{i} a_{i z}\right) z+\sum\left(w_{i}\right) t=\sum\left(w_{i} F_{i}\right)
\end{aligned}
$$

The normal equations are solved and the matrix $\mathbf{M}$ of their coefficients is inverted by the Crout method (Friedman and Foote, 1955).

The variance of the solution is

$$
S=\sqrt{\frac{\sum w_{i}\left(P_{i}-[P-O]_{i}-O\right)^{2}}{N-5}}
$$

where $N=$ number of stations. 
The standard errors of the rectangular coordinates of the source and the origin time are obtained from the variance and the diagonal elements of the inverse matrix.

$$
\sigma_{j}=S\left[M_{j j}{ }^{-1}\right]^{\frac{1}{2}} \quad j=x, y, z, t
$$

These values are typed out in kilometers for the coordinates and seconds for the origin time.

\section{Transformation of Coordinates}

The Clarke spheroid (Anon., 1952; Simmons, 1949) is taken as the basis of the geographic coordinates $(\sigma, \lambda, h)$ of stations and sources. Transformation to or from

TABLE 1

\begin{tabular}{c|c|c|c}
\hline Phase & $a_{i x}$ & $a^{a_{i y}}$ & $a_{i z}$ \\
\hline$P_{1}(\bar{P})$ & $\frac{1}{V_{1}} \frac{X-X_{i}}{D_{i}}$ & $\frac{1}{V_{1}} \frac{Y-Y_{i}}{D_{i}}$ & $\frac{1}{V_{\mathrm{I}}} \frac{Z-Z_{i}}{D_{i}}$ \\
$P_{2}\left(P_{n}\right)$ & $\frac{1}{V_{2}} \frac{X-X_{i}}{\Delta_{i}}$ & $\frac{1}{V_{2}} \frac{Y-Y_{i}}{\Delta_{i}}$ & $C_{2}$ \\
$P_{3}\left(P_{n}\right)$ & $\frac{1}{V_{3}} \frac{X-X_{i}}{\Delta_{i}}$ & $C_{3}$ & $\frac{Y-Y_{i}}{\Delta_{i}}$ \\
\hline
\end{tabular}

$D_{i}^{2}=\left(X-X_{i}\right)^{2}+\left(Y-Y_{i}\right)^{2}+\left(Z-Z_{i}\right)^{2}$

$\Delta_{i}^{2}=\left(X-X_{i}\right)^{2}+\left(Y-Y_{i}\right)^{2}+\left(Z-h-Z_{i}\right)^{2}$

the rectangular coordinate system is made through an intermediate system of spherical coordinates $\left(\varphi^{\prime}, \lambda^{\prime}, \rho^{\prime}\right)$. The surface of the spheroid is mapped on a sphere of radius $R$ tangent to the spheroid at $35^{\circ} \mathrm{N}, 118^{\circ} \mathrm{W}$, with its polar axis parallel to that of the spheroid. Mapping makes use of the equations

$$
\begin{aligned}
\varphi^{\prime} & =\left(35+\frac{180}{\pi} \frac{M(\varphi)}{R}\right)^{\circ} \\
\lambda^{\prime}-118^{\circ} & =\left(\lambda-118^{\circ}\right) N / R \\
\rho^{\prime} & =R-h
\end{aligned}
$$

where

$M(\varphi)=$ meridianal distance north of $35^{\circ} \mathrm{N}$ on the spheroid,

$R=$ radius of curvature of the spheroid in the meridian plane at $35^{\circ} \mathrm{N}$,

$N=$ radius of curvature of the spheroid in a vertical plane perpendicular to the meridian at $35^{\circ} \mathrm{N}$.

The shift of position of a point produced by this mapping varies as the square of the distance from $35^{\circ} \mathrm{N}, 118^{\circ} \mathrm{W}$ and is approximately $0.2 \mathrm{~km}$ at $30^{\circ}$ or $40^{\circ} \mathrm{N}, 113^{\circ}$ or $123^{\circ} \mathrm{W}$ : points which mark the boundary of the area for which this program was 
designed. Transformation between $\left(\varphi^{\prime}, \lambda^{\prime}, \rho^{\prime}\right)$ and $(X, Y, Z)$ is exact within the limits of the double-precision computation used in the program.

\section{Test on KNOwn Source}

A large quarry blast, 1951 March 31, near Corona, California, was timed by a field crew from the Seismological Laboratory, and affords data for an accurately known source and origin time (Gutenberg, 1952). Calculation with this program using arrival times of $P$ at nine stations gives the following results:

\begin{tabular}{l|c|c|c}
\hline & Observed & Calculated & Standard Error \\
\hline Origin time & $30^{\mathrm{m}} 33.4^{\mathrm{s}}$ & $30^{\mathrm{m}} 33.579^{\mathrm{s}}$ & $0.150^{\mathrm{s}}$ \\
Latitude & $33^{\circ} 50.81^{\prime} \mathrm{N}$ & $33^{\circ} 50.285^{\prime} \mathrm{N}$ & $1.422 \mathrm{~km}$ \\
Longitude & $117^{\circ} 30.36^{\prime} \mathrm{W}$ & $117^{\circ} 29.564^{\prime} \mathrm{W}$ & $1.643 \mathrm{~km}$ \\
Depth & 0 & $0.516 \mathrm{~km}$ & $2.764 \mathrm{~km}$ \\
\hline
\end{tabular}

\section{Limitations}

Bolt has pointed out that for highest accuracy in location of teleseisms it is necessary that the recording stations be uniformly distributed in azimuth about the epicenter, and that within each quadrant in azimuth the stations should be evenly distributed by distance. These conditions hold with some modification for local earthquakes. The first condition affects the accuracy of location of the epicenter; the second, the determination of depth of focus.

Since the partial derivatives of the travel times $\left(P_{2}-O\right)$ and $\left(P_{3}-O\right)$ with respect to depth are not dependent on epicentral distance, it is impossible to solve for both depth and origin time on the basis of a set of readings of $P_{2}$ or of $P_{3}$ alone. The fact that the two derivatives differ by less than ten per cent for the assumed structure raises serious doubts that a combination of $P_{2}$ and $P_{3}$ readings will furnish the basis for a reliable determination of depth and origin time.

This leaves three alternatives. First, at least one arrival time for $P_{1}$, and preferably more, may be included in the data. This condition has been achieved for sequences of aftershocks of large local earthquakes by operating temporary recording stations near the epicenter of the main shock.

Second, it may be possible to add to the program so that times of arrival of $S$ phases may be used in the least-square solution. The use of reliable $S$ times would impose restraints on the origin time and would increase by about fifty per cent the range of values of the partial derivatives of travel-time with respect to depth. However, it is often difficult to tell which $S$ phase appears on a given seismogram, and this uncertainty in identification must be considered in assessing the advantages gained by using times of $S$.

Finally, the present program usually gives an epicenter which changes very little after the first few iterations, together with an origin time corresponding to some calculated depth of focus. With this information, it is possible to compute either the origin time for an assumed depth of focus, or a depth of focus corresponding to the origin time which best fits the arrival times of all phases recorded at the most dependable station or stations in any particular case. 
John K. Gardner, research assistant at the Seismological Laboratory, has developed a matrix inversion routine that is more efficient in space and computing time than that in the program described here. He is cooperating with the author in revising this program to permit

(1) use of data from a larger number of stations,

(2) use of up to two $P$ and two $S$ arrival times for each station, with separate identification of each of the phases,

(3) computation of arrival times for sources below the Conrad discontinuity, and

(4) exclusion from the normal equations of data which give large residuals, the threshold of acceptability being lowered after each iteration of the least square solution for corrections.

\section{ACKNOWLEDGMENTS}

This research was supported by Contract No. AF-49 (638) 910 of the Air Force Technical Applications Center as part of the Advanced Research Projects Agency project VELA.

\section{REFERENCES}

Anonymous

1952. Formulas and Tables for the Computation of Geodetic Positions, U. S. C. G. S. Special Publication No. 8, 7th ed., 101 pp.

Bolt, B. A.

1960. "The Revision of Earthquake Epicentres, Focal Depths and Origin-times Using a High-speed Computer," Geophys. J., 3: 433-440.

Flinn, E. A.

1960. "Local Earthquake Location with an Electronic Computer," Bull. Seism. Soc. Am., $50: 467-470$.

Friedman, J., and R. J. Foote

1955. Computational Methods for Handling Systems of Simultaneous Equations, U. S. Dept. of Agriculture Handbook No. 94, 109 pp.

Gutenberg, B.

1952. "Waves from Blasts Recorded in Southern California," Trans. Am. Geophys. Union, 33: $427-431$.

Press, F.

1960. "Crustal Structure in the California-Nevada Region," J. Geophys. Res., 65: 1039-1051. Simmons, L. G.

1949. Natural Tables for the Computation of Geodetic Positions, Clarke Spheroid of 1866 , U. S. C. G. S. Special Publication No. 241, 86 pp.

Contribution No. 1056 ,

Division of Geological Sciences,

California Institute of Technology,

Pasadena, Califoria

Manuscript received October 18, 1961. 